\title{
ON THE CORRESPONDENCE BETWEEN SPACE SEXTIC CURVES AND PLANE QUARTICS IN FOUR-SPACE*
}

\author{
BY B. C. WONG
}

This paper proposes to show by means of an involutorial quartic transformation in space of four dimensions a certain correspondence between certain space sextics and plane quartics. The transformation is effected by four quadric varieties. $\dagger$ To a point is made to correspond the intersection of its polar spaces with respect to the quadric varieties. If a point describes a line, a plane, or a 3-space, the corresponding point describes a quartic curve, a two-dimensional surface of order 6 , or a three-dimensional variety of order 4 , respectively. The locus of points which transform into lines is the surface $J_{2}^{10}$ (of dimension 2 and degree 10) and the locus of these lines is $J_{3}^{15}$ (of dimension 3 and degree 15). The $J_{3}^{15}$ is the Jacobian of the $\left|M_{3}^{4}\right|$, images of the $S_{3}$ of $S_{4}$. The former is the fourfold two-dimensional surface on the latter and the latter is generated by the quadri-secants of the former.

Consider a fixed plane $\sigma_{2}$. It has 10 points $P$ in common with $J_{2}^{10}$. The surface $\Sigma_{2}^{6}$ into which $\sigma_{2}$ transforms is intersected by a 3 -space $S_{3}$ in a sextic curve $\Gamma_{1}^{6}$. The quartic variety $S_{3}^{4}$ corresponding to $S_{3}$ is met by $\sigma_{2}$ in a quartic $\gamma_{1}^{4}$ through $P$. The transform of $\gamma_{1}^{4}$ is a degenerate curve of the 16 th degree in 4 -space made up of 10 lines corresponding to the 10 points $P$ and the sextic $\Gamma_{1}^{6}$ which is in $S_{3}$. This sextic $\Gamma_{1}^{6}$ is said to correspond to the plane quartic $\gamma_{1}^{4}$.

The 10 points $P$ in $\sigma_{2}$ determine $\infty^{4}$ quartics; correspondingly, $\Sigma_{2}^{6}$ determines with the $\infty^{4} 3$-spaces in 4 -space $\infty^{4}$ sex-

* Presented to the Society, San Francisco Section, April 3, 1926.

$\dagger N$. Alderton, Involutory quartic transformation in space of four dimensions, University of California Publications in Mathematics, vol. 1 , No. 15 , pp. 345-358. 
tic curves. Two 3 -spaces $S_{3}$ and $S_{3}{ }^{\prime}$ transform into two hyperquartic surfaces $S_{3}^{4}$ and $S_{3}^{4 \prime}$ which determine with $\sigma_{2}$ two quartics $\gamma_{1}^{4}$ and $\gamma_{1}^{4 \prime}$ through the 10 points $P$ and 6 other points $Q$. The points $Q$ are, in fact, the intersections of $\sigma_{2}$ and the transform $\Sigma_{2}^{6 \prime}$ of the plane $\sigma_{2}^{\prime}$ determined by $S_{3}$ and $S_{3}^{\prime}$. These 16 points $P$ and $Q$ form the basis of a pencil of quartic curves; correspondingly, the plane $\sigma_{2}^{\prime}$ is the basis of a pencil of 3spaces each containing a sextic curve.

Of all the quartics through the points $P$ in $\sigma_{2}$ some are unicursal and others are of deficiency 1,2,3. Suppose $\gamma_{1}^{4}$ is trinodal. Then the corresponding $\Gamma_{1}^{6}$ has three real (as distinguished from "apparent") double points and is the intersection of $\Sigma_{2}^{6}$ and a triply tangent space $S_{3}$. The number of apparent double points is 7 . Similarly, a 3 -space which is simply-, or doubly-tangent to $\Sigma_{2}^{6}$ gives rise to a sextic with one, or two nodes corresponding to a quartic in $\sigma_{2}$ with one, or two nodes respectively.

Among the quartics through $P$ in $\sigma_{2}$ there are many degenerate ones, and these transform into degenerate space sextics. Of interest are those made up of pairs of conics, each pair having one component through five points of $P$ and the other through the remaining five. The sextic corresponding to such a degenerate quartic is composed of two space cubics having in common four points which are the transforms of the four points of intersection of the two conics. Its space is quadruply tangent to $\Sigma_{2}^{6}$. Through the 10 points $P, 252$ conics can be drawn and they form 126 pairs of degenerate quartics of this type. Hence there are 1263 -spaces which are quadruply tangent to $\Sigma_{2}^{6}$, each containing a $\Gamma_{1}^{6}$ composed of two twisted cubics with four points in common.

It is to be noted that there are other quadruply tangent 3 -spaces but they intersect $\Sigma_{2}^{6}$ in different types of degenerate sextics. For example, a quartic in $\sigma_{2}$ composed of a line through two points of $P$ and a nodal cubic through the remaining eight gives rise to a sextic whose components are a conic and a twisted quartic with a real double point having three points in common. These three common points and the node on the 
quartic are the four points of contact between the 3-space containing the sextic and $\Sigma_{2}^{6}$. Or, it may happen that 6 of the 10 points $P$ lie on a conic $\kappa^{2}$. This conic $\kappa^{2}$ and any conic $\kappa^{2 \prime}$ through the remaining 4 points of $P$ form a degenerate quartic whose transform is a degenerate sextic made up of a conic $K_{1}^{2}$ and a quartic $K_{1}^{4}$ having four points in common. If $\kappa^{2 \prime}$ is a line pair, then the quartic $K_{1}^{4}$ decomposes into a pair of conics with one point in common. Each of these two component conics has two points in common with $K_{1}^{2}$. The sextic is now composed of three conics and its space is quintuply tangent to $\Sigma_{2}^{6}$.

Now suppose the 3-space $S_{3}$ contains the plane $\sigma_{2}$. It intersects its own transform, $M_{3}^{4}$, in a quartic surface $J_{2}^{4} ; J_{2}^{4}$ is the Jacobian quartic surface of the four quadric surfaces in which $S_{3}$ meets the four quadric varieties used to effect the transformation. The transform $\Sigma_{2}^{6}$ of $\sigma_{2}$ meets $S_{3}$ in the sextic whose points are in one to one correspondence with the quartic $\gamma_{1}^{4}$ common to $\sigma_{2}$ and $J_{2}^{4}$. The quartic $\gamma_{1}^{4}$ passes through the 10 points $P$ and the 6 points $Q$ which are on $\Sigma_{2}^{6}$ and therefore on the sextic $\Gamma_{1}^{6}$. As $\sigma_{2}$ varies in $S_{3}, \Gamma_{1}^{6}$ varies on $J_{2}^{4}$. Corresponding to the $\infty^{3}$ plane sections of $J_{2}^{4}$ there are $\infty^{3}$ sextics on $J_{2}^{4}{ }^{*}$ Any pencil of planes in $S_{3}$ gives rise to a pencil of sextics through four points which correspond to the four points in which the axis of the pencil of planes meets $J_{2}^{4}$. A further discussion of this configuration is unnecessary as it is a configuration in a three-dimensional space and is well known. $\dagger$

The University of California

* The totality of sextics on $J_{2}^{4}$ is $\infty^{6}$ but only $\infty^{3}$ of them correspond to plane quartics whose planes lie within $S_{3}$ and the others correspond to plane quartics whose planes are not contained in $S_{3}$.

$\dagger$ Jessop, Quartic Surfaces, Cambridge Press, 1916, Chapter IX. 